九州大学学術情報リポジトリ

Kyushu University Institutional Repository

\title{
Sputter Erosion Sensor for Anode Layer Type Hall Thrusters Using Cavity Ring-Down Spectroscopy
}

\section{Yamamoto, Naoji}

Department of Advanced Energy Engineering Science, Kyushu University : Assistant Professor

Tao, Le i

Department of Mechanical Engineering, Colorado State University

Rubin, Binyamin

Department of Mechanical Engineering, Colorado State University

Williams, John D.

Department of Mechanical Engineering, Colorado State University

他

http://hdl. handle. net/2324/16992

出版情報: Journal of propulsion and power. 26 (1), pp.142-148, 2010-01. American Institute of Aeronautics and Astronautics

バージョン :

権利関係 : 


\title{
Sputter Erosion Sensor for Anode Layer Type Hall Thrusters Using Cavity Ring-Down Spectroscopy
}

\author{
Naoji Yamamoto ${ }^{1}$ \\ Graduate School of Engineering Sciences, Kyushu University, Kasuga, Fukuoka 816-8580, Japan \\ Lei Tao ${ }^{2}$, Binyamin Rubin ${ }^{3}$, John D. Williams ${ }^{4}$, and Azer P. Yalin ${ }^{5}$ \\ Department of Mechanical Engineering, Colorado State University, Fort Collins, USA
}

\begin{abstract}
We report the development of a sputter erosion monitoring system to study Hall thruster lifetime and contamination. The laser-based sensor uses the continuous-wave cavity ring-down spectroscopy (cw-CRDS) technique and allows for in situ measurements in near real-time. The cw-CRDS diagnostic allows direct probing of sputter products in their ground state, thereby providing a reliable quantitative measure of their overall number density. Combining the number density of sputtered particles with their velocity allows determination of the flux of sputtered particles and erosion rate. We perform proof of principle experiments in which sputtered manganese (Mn) atoms from the acceleration channel of an anode layer type Hall thruster are measured. The measurement strategy is to detect the Mn atoms via an absorption line from the ground state at a wavelength of $403.076 \mathrm{~nm}$ (air). The measured path-integrated number density of sputtered $\mathrm{Mn}$ atoms is $1.7 \pm 0.3 \times 10^{13} \mathrm{~m}^{-2}$ for an argon anode mass flow rate of $2.08 \mathrm{mg} / \mathrm{s}$ and discharge voltage of $250 \mathrm{~V}$. A finite element sputter model is used to compare the CRDS results against validating mass loss measurements and shows good agreement.
\end{abstract}

\section{Nomenclature}

$\begin{array}{lll}A_{k i} & = & \text { Einstein A coefficient, } 1 / \mathrm{s} \\ A b s & = & \text { Absorbance } \\ c & = & \text { Speed of light, } 2.998 \times 10^{8} \mathrm{~m} / \mathrm{s} \\ E_{i} & = & \text { Energy of state } i, \mathrm{~J} \\ E_{k} & = & \text { Energy of state } k, \mathrm{~J} \\ E_{b} & = & \text { Binding energy, } \mathrm{J} \\ g_{i} & = & \text { Degeneracy of state } i \\ g_{k} & = & \text { Degeneracy of state } k \\ k & = & \text { Absorption coefficient, } \mathrm{m}^{-1} \\ l & = & \text { Length of the ring-down cavity, } \mathrm{m} \\ l_{\mathrm{abs}} & = & \text { Path length of absorbing species, } \mathrm{m} \\ N_{i} & = & \text { Lower state concentration, } \mathrm{m}^{-3}\end{array}$

\footnotetext{
${ }^{1}$ Assistant Professor, Department of Advanced Energy Engineering Science, 6-1 Kasuga-kouen, Kasuga, Fukuoka 816-8580, Japan, yamamoto@aees.kyushu-u.ac.jp and Member AIAA.

2 Doctoral candidate, Department of Mechanical Engineering, Colorado State University, Fort Collins, CO, 80523-1320,USA, leitao@lamar.acns.colostate.edu.

3 Post-Doctoral Researcher, Department of Mechanical Engineering, Colorado State University, Fort Collins, CO, 80523-1374, USA, brubin@engr.colostate.edu and Member AIAA.

4 Assistant Professor, Department of Mechanical Engineering, Colorado State University, Fort Collins, CO, 80523-1374, USA, johnw@engr.colostate.edu and Member AIAA.

${ }^{5}$ Associate Professor, Department of Mechanical Engineering, Colorado State University, Fort Collins, CO, 80523-1374, USA, azer.yalin@colostate.edu and Member AIAA.
} 


$\begin{array}{lll}R & = & \text { Mirror reflectivity } \\ S(t, v) & = & \text { Ring-down signal as function of time and laser frequency } \\ S_{0} & = & \text { Initial ring-down signal } \\ x & = & \text { Position along the optical axis, } \mathrm{m} \\ v & = & \text { Laser frequency, Hz } \\ v_{k i} & = & \text { Resonant frequency of transition, } \mathrm{Hz} \\ \tau & = & \text { Ring-down time, } \mathrm{s} \\ \tau_{0} & = & \text { Empty cavity ring-down time, } \mathrm{s}\end{array}$

\section{Introduction}

$1_{\text {[1-3]. They offer an attractive combination of high thrust efficiency (exceeding } 50 \%) \text { and specific impulse }(\sim 1,000-3,000}^{\text {all thrusters are a class of electric propulsion device in which a propellant gas is ionized and accelerated to produce thrust }}$ s). In comparison to chemical rockets, the high specific impulse is attractive for large delta V missions such as satellite positioning and station-keeping, and space exploration. Hall thrusters also have a higher thrust density than ion thrusters due to the existence of electrons in the ion acceleration zone. In addition, the lack of grids is attractive owing to the potential for reduced failures. Since the 1970s over 200 Hall thrusters have been operated in space. A key requirement for the practical use of Hall thrusters is the ability to operate for long durations, for example a Hall thruster used for north-south station keeping (NSSK) of a commercial spacecraft will have to operate for over 5,000 hours over the course of its mission [4, 5]. The primary life-limiter for Hall thrusters is acceleration channel wall erosion [6, 7]. A thruster is generally considered to have reached end of life when the channel is fully eroded and the underlying magnetic yoke becomes exposed. The physical mechanism causing the erosion is sputtering of the channel material due to bombardment by energetic particles, primarily propellant ions having undergone radial acceleration. In addition to causing channel erosion and associated lifetime concerns, sputtered particles can redeposit on spacecraft components such as solar-arrays, thereby degrading their performance and potentially compromising spacecraft operation.

The problems of lifetime and redeposition may be addressed with both numerical modeling and experimental measurement. Research in the former area is underway but the fidelity of current models [8-10] tends to be limited by the accuracy in modeling the fluxes of ions to the channel walls (angular and energy distributions) and lack of knowledge of the sputter yields of the wall channel materials (especially at the needed low ion energies). Experimental lifetime measurements are also challenging. With proposed thrust durations now as long as 5-10+ years, ground-based life tests over the full thruster duration are becoming increasingly expensive and limiting in terms of technology insertion schedules. And, even when such tests are performed, it is hard to infer the effect of varying thruster operating parameters such as voltage and mass flow rate. What is needed, therefore, is a method of quantitatively measuring thruster erosion rates non-intrusively in real- or near real-time, for example by in situ measurement of the eroded wall material as we demonstrate here. Such measurements 
would complement numerical modeling approaches and would be dramatically faster (and cheaper) than full life testing, while also allowing study of how operating conditions and thruster design changes affect erosion.

A partial list of non-optical techniques for sputter measurements include weight loss [11], collector plates [12], quartz crystal microbalance [13, 14], radioactive tracers [15], mass spectrometer [16], and Rutherford backscattering [17]. The full life testing referred to above has generally employed weight loss or profilometry, neither of which is generally suitable for (near) real-time measurements. Optical techniques including optical emission spectroscopy (OES) $[18,19]$, laser induced fluorescence (LIF) [20] and multi-photon ionization coupled to a time of flight mass spectrometer [21, 22] have been used for species-specific sputtering measurements. OES has also been investigated for sputter erosion thruster diagnostics as is discussed below. The use of LIF has proven to be effective for velocity measurement though challenging for quantitative number density. L Laser absorption spectroscopy (LAS) measurements can be considered, but previous research on diode laser absorption in an anode layer type Hall didn’t yield sufficient sensitivity [7]. The approach presented here uses cavity ring-down spectroscopy (CRDS), a technique which is also based on LAS but which provides significantly improved sensitivity through the use of an optical cavity. Past work has developed and validated CRDS measurements of sputtered refractory metal and boron nitride targets exposed to ion beams [23-28]. Here, we show for the first time that the method can be extended to a CRDS erosion sensor for Hall thrusters, thereby providing a new method for thruster development and life-testing.

As mentioned above, OES has also been developed as a diagnostic method for sputter erosion studies of thrusters [29-35]. Like CRDS, OES can provide in situ measurements in near real-time. In terms of quantifying the sputter erosion, the most critical difference is that OES measures optical emission and therefore probes upper (excited) states, while CRDS is a form of LAS and therefore measures lower states including ground states. Of course, for measurements of thruster erosion the goal is to measure the overall population of sputtered species, not the population of a specific state. For interpretation of optical emission signals, population distributions are generally found from collisional-radiative models or simplifying assumptions based on such models. Corona models are generally used in which it is assumed that population fractions of excited and ionized levels are due to a balance between collisional excitation and spontaneous radiative decay, and the plasma is assumed to be optically thin [36]. An approach to determine sputter product number density has been to attempt to normalize away the dependence on rate constants $\left(T_{e}\right.$ and $n_{e}$ ) by considering ratios of emission intensities from lines (upper-state levels) having similar rate constant dependences, for example by looking at the ratio of emission from a sputter product to emission of neutral xenon [30], or ratio of emission from a sputter product to emission of xenon ions [31], or ratio of product of sputter product emission with neutral Xe emission to ionic Xe emission [32]. Although, the relative simplicity and ease of implementing OES are attractive, the challenges of signal interpretation can introduce doubt in the quantification. In 
comparison, CRDS provides more direct quantification since measuring ground states probes a high fraction of the overall population that is relatively insensitive to changes in rate constants. However, in regions of strong excitation, as may occur in the ionization region of the Hall thruster, depletion of the ground state population to excited or ionized states should be considered (as is discussed in Section IV for the current experiment). Additionally, absolute quantification of number densities in CRDS is far simpler as the technique is self-referencing, while OES requires external calibration so that typically only relative measurements are made. Finally, we note that both OES and CRDS directly measure number densities, while determination of erosion rates require particle fluxes, i.e. product of number density and velocity. The simplest approach to determine flux is to assume that the velocity (energy) distribution of sputter products follows a Thomson distribution, though complementary measurements, for example from LIF or CRDS lineshapes, or modeling results could also be used.

The layout of the remainder of the paper is as follows. Section II of the paper describes the nature of the CRDS technique and its sensitivity. Section III presents the experimental setup used for the CRDS sensor and operation of the

anode layer type Hall thruster. For this class of thruster the acceleration channel is generally formed from a conductive material [37-39] and our proof of principle experiments use a stainless steel channel. Erosion was measured by probing sputtered manganese atoms (contained within the stainless steel). For optimum sensitivity we have implemented CRDS with a continuous-wave (cw) laser as opposed to a pulsed laser source. Differences of CRDS with cw versus pulsed lasers are discussed in Section II. The experimental results and discussion are presented in Section IV. The sensor was used to measure the path-integrated concentration of sputtered manganese at different thruster operating conditions. The CRDS results were validated against mass-loss measurements. Relating the CRDS number density measurements to the mass-loss requires consideration of the flux of the sputtered particles, for which we have used as simple finite element model. Finally, conclusions are presented in Section V.

\section{Cavity Ring-Down Spectroscopy (CRDS)}

CRDS is a path-enhanced laser absorption method that provides extremely high-sensitivity. The technique is seeing growing use in a range of applications including molecular spectroscopy, atmospheric monitoring, combustion diagnostics, and plasma diagnostics and has been described in several review articles [40-42]. The technique can readily measure per-pass optical absorbance of $<0.1$ ppm for measurement times of seconds. (The optical absorbance is the product of absorption coefficient and path length, and in the limit of small absorbance corresponds to the fractional amount of light absorbed.) Our past work has developed the use of CRDS for the study of sputtered particles in electric propulsion applications [23-28]. As 
mentioned above, the technique is quantitative, self-referencing, and can measure ground states.

As shown in Fig. 1, the basic idea is to house the absorbing species (i.e. the sputtered atoms) within a high finesse optical cavity formed from high-reflectivity (HR) mirrors. The probe laser beam is coupled into the optical cavity where it "bounces" many times back-and-forth between the mirrors. Owing to the high reflectivity, the light within the cavity makes many passes within the cavity (e.g. $\sim 10^{4}$ passes for $\mathrm{R} \sim 0.9999$ ), so that the effective path length is greatly increased. A detector placed behind the cavity measures the temporal decay of optical intensity within the cavity. The difference in the temporal decay rate with and without the absorber (or with the laser tuned on/off the resonance) yields the sample concentration. The technique affords high sensitivity owing to a combination of long effective path length and insensitivity to laser energy fluctuations (since a rate is measured). CRDS can be implemented with either pulsed lasers or cw lasers [40-42]. The latter method, which we use, is termed cw-CRDS and typically provides higher absorption sensitivities owing to higher sampling rates and narrower laser linewidths (allowing coupling to only a single cavity mode). In cw-CRDS setups, the light injected to the cavity must first be extinguished to generate the ring-down signal. Details of our experimental configuration are described in Section III.



Fig. 1. Schematic diagram of CRDS technique.

Under typical conditions the ring-down signal $S(t, v)$ decays single exponentially versus time as[40-44]:

$$
\begin{aligned}
& S(t, v)=S_{0} \exp \left(-\frac{t}{\tau(v)}\right) \\
& \frac{1}{\tau(v)}=\frac{l}{c} \quad\left[\int k(x, v) d x+(1-R)\right]
\end{aligned}
$$

where $\tau$ is the 1/e time of the decay (termed the ring-down time), $c$ is the speed of light, $l$ is the cavity length, $k(x, v)$ is the absorption coefficient, $v$ is the laser frequency, $x$ is the position along the optical axis, and 1- $R$ is the effective mirror loss (including scattering and all cavity losses). If the absorber is uniformly present over a column length $l_{a b s}$, then $\int k(x, v) d x$ can be replaced with the product $k(v) l_{a b s}$. The standard procedure is to measure the ring-down signal and then fit with an exponential to find the ring-down time $\tau$. Combining $\tau$ with the "empty cavity ring-down time" $\tau_{0}$ (measured by detuning the laser) allows determination of the sample absorbance, $A b s(v)$, and absorption coefficient: 


$$
\operatorname{Abs}(v)=\int k(x, v) d x=\frac{l}{c}\left[\frac{1}{\tau}-\frac{1}{\tau_{0}}\right]
$$

A commonly used approach is to scan the laser frequency across an absorption line and to measure the wavelength- (or frequency-) integrated spectrum, i.e. the line area. Assuming the spectroscopic line parameters are known, the measured line area $\int A b s(v) d v$ of a transition from lower state $i$ to upper state $k$ can be readily converted to the path-integrated concentration of the lower state $\int N_{i} d x$ as:

$$
\int N_{i} \mathrm{~d} x=\frac{v_{k i}{ }^{2}}{c^{2}} \frac{8 \pi}{A_{k i}} \frac{g_{i}}{g_{k}}\left(\int A b s(v) d v\right)
$$

For cases where the spatial distribution of particles is non-uniform, concentration profiles can be determined in several ways. For rough approximation one can assume a uniform concentration profile over a known column length, $l_{a b s}$. Alternatively, Abel inversion or other inversion approaches based on inversion and modeled spatial profiles can be used [28, 45]. CRDS can also be used to extract velocity information from the measured spectral lineshapes [26, 28]. In this work, we measure the path-integrated concentration and a finite element model is used to examine the particle flux, erosion rate, and mass-loss.

\section{Experimental Setup}

Figure 2 shows a cross-section of the $1 \mathrm{~kW}$ class anode layer type Hall thruster used in the current experiments [46]. The inner and outer diameters of the acceleration channel are $48 \mathrm{~mm}$ and $72 \mathrm{~mm}$ respectively. An inner solenoid coil and four outer solenoid coils create a predominantly radial magnetic field in the acceleration channel. The magnetic field distribution along the channel median is almost uniform in the short acceleration channel. Magnetic flux density is higher on the inner wall and decreases with radius, since the magnetic flux between the poles is conserved. In these experiments, the acceleration channel is made from stainless steel (SUS316). The SUS 316 contains about $1 \%$ manganese $(0.81 \%$ from manufacturer's datasheet) and the CRDS measurements are based on detection of the manganese sputtered from the steel. The separation between the guard ring and anode is $1 \mathrm{~mm}$. The thruster has a hollow annular anode, which consists of two cylindrical rings, with a propellant gas fed through them. The width of the hollow anode is $8 \mathrm{~mm}$, and the gap between the tip of the anode and the exit of the acceleration channel is fixed at $3 \mathrm{~mm}$. High-purity (99.999\%) argon gas was used as the propellant with thermal mass flow controllers (Brooks 5850E). A hollow cathode is used as the electron source. Thruster operating conditions are specified in Section IV. 


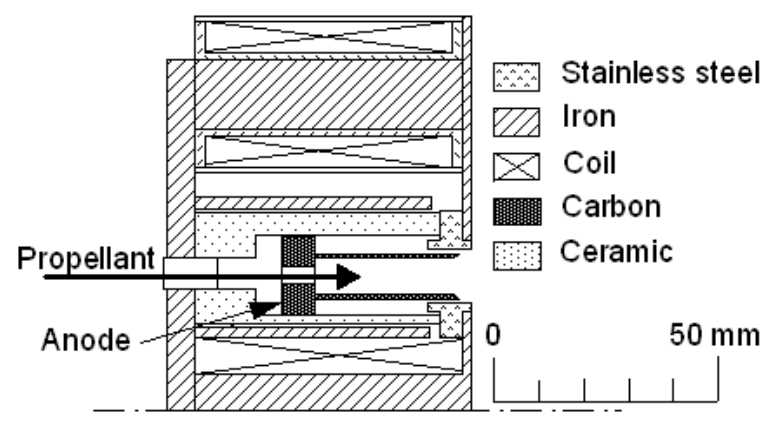

Fig. 2. Cross-section of the anode layer type Hall thruster.

Tests are conducted in a vacuum chamber of $1.5 \mathrm{~m}$ diameter by $4.6 \mathrm{~m}$ length. The pumping system includes a dry mechanical pump (Edwards GV250) assisted by a mechanical booster pump (Edwards EH-1200) and two diffusion pumps (Varian HS-20) with overall pumping speed of $35000 \mathrm{l} / \mathrm{s}$ for air. The chamber baseline pressure is below $7 \times 10^{-4} \mathrm{~Pa}$. Any contribution of sputtered manganese from the chamber wall is negligible owing to positioning of the Hall thruster.

Figure 3 shows a diagram of the experimental setup emphasizing the CRDS. We use a tunable diode laser with external cavity (ECDL) to measure the transition line of manganese at $403.076 \mathrm{~nm}$ (air). The transition data for this measurement are shown in Table 1 (from National Institute of Standards and Technology database). The modehop free tuning range of the laser is about $30 \mathrm{GHz}$ and the laser linewidth is less than $5 \mathrm{MHz}$. The laser output power is $\sim 5 \mathrm{~mW}$ with a power of $\sim 1 \mathrm{~mW}$ injected into the optical cavity. The diode laser is set for a $25 \mathrm{GHz}$ mode-hop-free sawtooth frequency scan every 0.5 seconds (up- and down- scan in $1 \mathrm{~s}$ ). An optical isolator (within the laser head) is used to prevent back reflections into the laser and a polarization maintaining (PM) single mode fiber is used to deliver the beam to the inside of the vacuum chamber (using a custom feed-through). The fiber output, mode-matching and delivery optics (not shown in Fig. 3), and optical cavity are held on an optical rail system within the chamber. The rail is heat-shielded and uses low thermal-expansion rods to minimize changes in cavity alignment caused by the heat flux from the thruster. Rubber damping mounts are used to minimize vibration effects. Because any optical loss within the cavity reduces the ring-down time and sensitivity, it is not possible to mount the mirrors external to the vacuum chamber and to use standard windows or viewing ports. It is possible to mount the windows on the chamber walls and use them also to seal the vacuum [23,24], but, especially for large facilities where the chamber may deform owing to pump-down or thermal reasons, we view that it is preferable to use a fiber coupled internal rail-system for the cavity. Such a system is easier to align (and alignment can be done independently of the vacuum chamber) and is portable so that it can be implemented in different vacuum chamber test facilities with minimal modification. Finally, it can be easily mounted on a translation state for spatial scanning. 


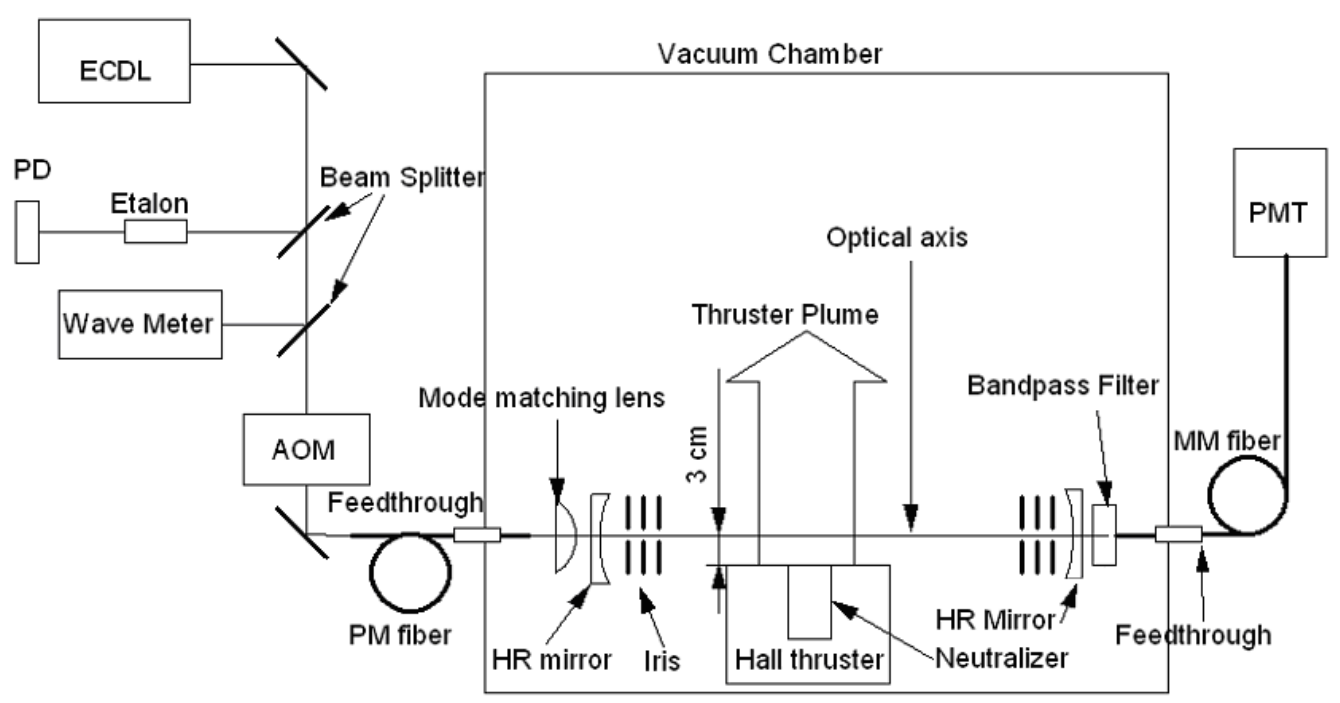

Fig. 3. Schematic diagram of CRDS sputter erosion monitoring system.

Table 1. Transition data for manganese line.

\begin{tabular}{ll}
\hline \hline$\lambda, \mathrm{nm}$ (Air) & 403.076 \\
\hline$E_{i}, \mathrm{eV}$ & 0 \\
$E_{k}, \mathrm{eV}$ & 3.075 \\
$A_{k i,} \mathrm{~s}^{-1}$ & $1.7 \times 10^{7}$ \\
$g_{i}$ & 6 \\
$g_{k}$ & 8 \\
\hline \hline
\end{tabular}

An aspheric lens is used at the fiber exit to (approximately) collimate the beam and to match the beam to the $\mathrm{TEM}_{00}$ mode of the cavity. The optical cavity is $0.70 \mathrm{~m}$ in length and is formed by a pair of high reflectivity mirrors $25.4 \mathrm{~mm}$ in diameter with radius-of-curvature of $1 \mathrm{~m}$. We typically operate with empty-cavity (detuned) ring-down times of $\sim 20 \mu$, corresponding to $\mathrm{R} \sim 99.99 \%$ (close to manufacturer’s specifications). A series of irises (diameter $\sim 2 \mathrm{~mm}$ ) held within a re-entrant tube are positioned in front of each cavity mirror to prevent deposition of sputtered particles and excess exposure to ultraviolet light, both of which can degrade mirror reflectivity. Mirror degradation owing to the former can be readily remedied by standard cleaning with methanol and lens tissue, while degradation owing to the latter naturally recovers after 
several hours of non-exposure. With our cavity setup including heat-shielding mentioned above, the cavity remains stable for experiment durations of $\sim 3-10$ hours which is sufficient for stable thruster operation and acquisition of multiple data points. After longer durations the baseline ring-down time and detection sensitivity are reduced. With future improvements in mirror shielding and cavity design, experimental durations of $>\sim 100$ hours should be possible without any user intervention. Light exiting the cavity is relayed via a multi-mode(MM) optical fiber to a photomultiplier tube (PMT) outside the vacuum chamber. A dielectric interference filter ( $40 \mathrm{~nm}$ band-pass) and an iris are used to suppress background light and emission from the plasma. Ring-down signals are measured with a $20 \mathrm{MHz}$ 12-bit analog-to-digital acquisition board connected to a personal computer and a custom Labview@ $@$ program is used for exponential fitting with the nonlinear Levenberg-Marquardt method.

Owing to the narrow laser linewidth (relative to the cavity free spectral range), we need to monitor for overlap of the laser frequency with the cavity modes and then extinguish the light to obtain ring-down signals. These tasks are accomplished with a threshold detection circuit and acousto-optic modulator (AOM). The combined time response of the threshold circuit and AOM firing is less than $400 \mathrm{~ns}$. A solid etalon (free spectral range $=2.26 \mathrm{GHz}$ ) is used as a frequency reference and a wave-meter is used for coarse frequency measurement. The positions of these components are shown in Figure 3. The setup is similar to that used in our past work on sputter monitoring and end-point for ion beam sputtering [47], though here the laser beam is fiber delivered in and out of the vacuum chamber and the optical cavity hardware is on a rail-system inside of the chamber.

\section{Results and Discussion}

The primary outcome of this research is demonstration of the ability to measure sputter eroded particles in the thruster plume in (near) real-time. Figure 4 shows the measured spectrum of sputtered manganese for thruster discharge voltage of $250 \mathrm{~V}$ and mass flow rate of $2.08 \mathrm{mg} / \mathrm{s}$. The CRDS measurements are shown with symbols (with $1 \mathrm{GHz}$ linearly averaged smoothing along the abscissa), the hyperfine structure [48] is shown with vertical bars, and a modeled lineshape (explained below) is shown as a solid curve. The optical axis is positioned $3 \mathrm{~cm}$ downstream of the exit plane and centered in the other directions. The path-integrated Mn number density is estimated using eqn.(3) from the wavelength-integrated area of the absorption line. The laser tuning range is insufficient to scan over the full line so we find the line area by numerically integrating half of the lineshape (the low-frequency side of the peak) and doubling. For this condition, the result is a path-integrated Mn concentration of $1.7 \pm 0.3 \times 10^{13} \mathrm{~m}^{-2}$ (where the uncertainty is partly due to the area fitting but dominated by the $18 \%$ uncertainty in $A_{k i}$ ). In principle the measured concentration corresponds to the directly measured ground state, but 
because there are no low-lying levels the ground state and overall Mn populations are equivalent (to within our uncertainty). More precisely, a Boltzmann analysis for a characteristic temperature of $1500 \mathrm{~K}$ shows that the ground state comprises $>99 \%$ of the overall population [24, 26]. Note that there is little reason to assume Boltzmann distributions for the sputtered particles, but past research has generally shown distributions similar to Boltzmann with "temperatures" generally in the range $\sim 500-1500 \mathrm{~K}$ (e.g., Reference 24 and therein).

As discussed in Section I, it is necessary to consider possible loss (depletion) of ground state atoms prior to their reaching the measurement location. If such loss is significant then it would reduce signal levels and also introduce systematic error (similar to the challenges of OES), since the measured atoms would constitute a somewhat unknown fraction of those that were originally sputtered. Simple estimates shows such effects to be minimal in the present experiment with a loss of $<4 \%$ of ground state sputtered particles to an ionized state (smaller than our uncertainty). The calculation conservatively assumes the sputtered particles move a distance of $5 \mathrm{~cm}$ through a region having $n_{e}=10^{11} \mathrm{~cm}^{-3}$ and $T_{e}=5 \mathrm{eV}$ [49] and uses calculated ionization cross-sections [50]. For the velocity of sputtered particles, an average from the Thomson distribution is used.

We have performed a study of dependence of sputter erosion on discharge voltage. Table 2 shows the sputtered manganese number density measured in the thruster plume for three different discharge voltages. The path-integrated number densities are determined from the CRDS as described above. For these conditions, the sputtered particle number density increases roughly linearly (or slightly more strongly) with discharge voltage. As a means of comparison we have also performed mass-loss tests of one-hour duration and these results are also shown in the table. The mass-loss values are found by measuring the mass change of the stainless steel channel at the corresponding condition (with uncertainty of $5 \%$ due to scale resolution and small changes in thruster operation conditions). The trend of mass loss versus discharge voltage is in broad agreement with that for the CRDS measurements of number density.

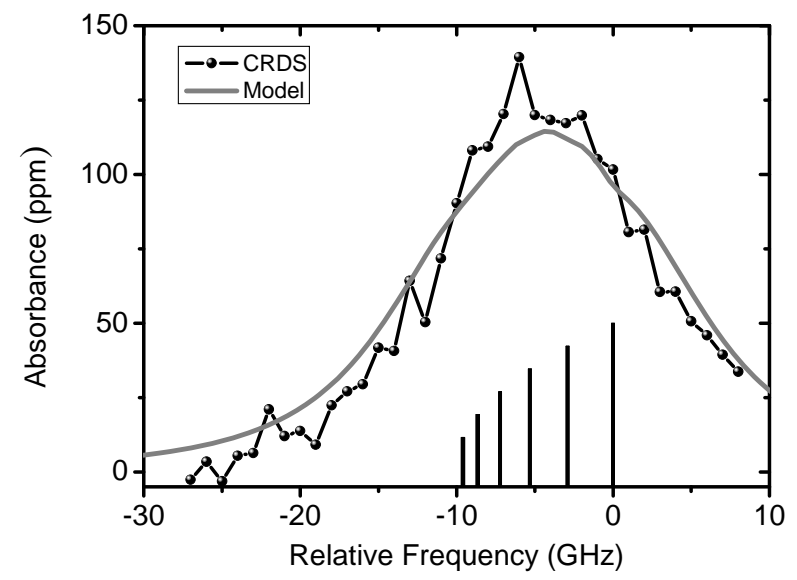

Fig.4 Spectrum of sputtered Mn measured by CRDS. 
Table 2. Dependence of sputtered particle number density and mass erosion rate on discharge voltage. Mass erosion rates are found from direct mass loss measurement and estimated from the CRDS (see text).

\begin{tabular}{cccc}
\hline \hline $\begin{array}{c}\text { Discharge } \\
\text { Voltage, V }\end{array}$ & $\begin{array}{c}\text { CRDS Number } \\
\text { Density, } \mathrm{m}^{-2}\end{array}$ & $\begin{array}{c}\text { Erosion rate, kg/hr } \\
\text { (from Mass Loss) }\end{array}$ & $\begin{array}{c}\text { Erosion rate, kg/hr } \\
\text { (from CRDS) }\end{array}$ \\
\hline 150 & $8.2 \pm 1.5 \times 10^{12}$ & $2.2 \pm 0.11 \times 10^{-4}$ & $2.0 \pm 0.8 \times 10^{-4}$ \\
200 & $1.4 \pm 0.3 \times 10^{13}$ & $3.6 \pm 0.09 \times 10^{-4}$ & $3.4 \pm 1.4 \times 10^{-4}$ \\
250 & $1.7 \pm 0.3 \times 10^{13}$ & $4.6 \pm 0.05 \times 10^{-4}$ & $4.1 \pm 1.6 \times 10^{-4}$ \\
\hline \hline
\end{tabular}

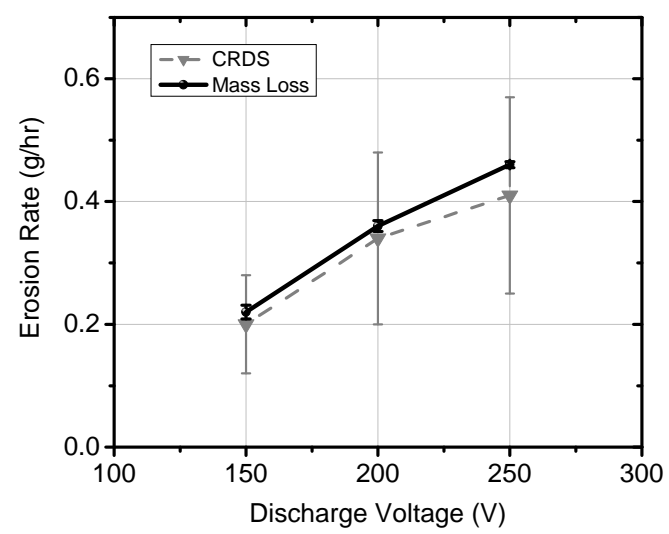

Fig.5. Mass erosion rates from found direct mass loss measurement and estimated from the CRDS sensor.

For quantitative comparison of the CRDS number density and measured mass-loss we use a finite element model for the sputtering [28]. In summary, we consider sputtering from four surfaces of the thruster: 1) an annulus of width 3 mm on the inner channel facing the inside of the thruster (with surface normal in the radial direction); 2) an annulus similar to 1) but on the outer channel; 3) an annulus of width $2 \mathrm{~mm}$ on the inner channel but facing downstream (with surface normal in the axial direction); and 4) an annulus similar to 3 but on the outer channel (see Fig. 5). Identification of these surfaces is based on visual inspection of the channel. Surfaces 1) and 2) are typical while 3) and 4) may be expected for this thruster configuration (with argon operation) since the ionization region appears to extend beyond the end of the channel. For coarse approximation we assume that the amount of sputtering per unit area from all four surfaces is equal (see below) and we set this to an arbitrary value. We assume that from each sputtering site particles are ejected with a diffuse sputter yield distribution, i.e. proportional to the cosine of the ejection angle relative to the surface normal, and we assume a Thomson distribution for particle velocity (energy) using the binding energy of $\mathrm{Mn}\left(E_{\mathrm{b}}=2.92 \mathrm{eV}\right)$ and $n=1.54$ as the exponent on the velocity distribution [28]. We make a series of elements on each sputtering surface and along the optical beam, and then 
find sputter contributions from each source location to each location along the optical axis. These contributions are added together to determine the path-integrated number density. We determine the corresponding expected mass-loss from the flux of particles from the four surfaces (neglecting redeposition). We assume that the composition of species sputtered from the steel mimics the composition of the bulk steel in order to find the total mass flux (of all species). In this way we can find the proportionality factor between the Mn number density along the optical axis (i.e. the CRDS measurement) and the associated total mass flux (mass erosion rate), and use this factor to determine mass erosion rates from the CRDS measurements. These CRDS-based erosion rates are also included in Table 2 and Figure 5 and are in good agreement with the direct mass-loss measurements, thereby providing a validation of the CRDS results. The uncertainty in the (CRDS) modeled erosion values $( \pm 40 \%$ ) are found from contributions due to possible variation of the sputter flux from each surface (we consider cases where each surface sputters at a rate of twice the others $( \pm 16 \%)$ ), changes from diffuse sputter yield to an over- or under-cosine profile $( \pm 5 \%)$, changing the exponent used in the Thomson distribution $( \pm 32 \%)$, and the uncertainty in corresponding CRDS number density measurement $( \pm 18 \%)$. The overall uncertainty in CRDS erosion rate is rather high but could be improved in several ways including more accurate determination of the $A_{k i}$ coefficient, spatial scanning with Abel inversion to lower the geometric contribution to the uncertainty, and better knowledge of the velocity distribution (e.g. by CRDS lineshape studies or complementary LIF measurements). The model also allows simulation of the absorption lineshape based on Doppler shifts [26,28]. A modeled lineshape computed with the above assumptions and including the hyperfine structure of Mn [39] is included in Figure 3 and is in reasonable agreement with the measured lineshape.

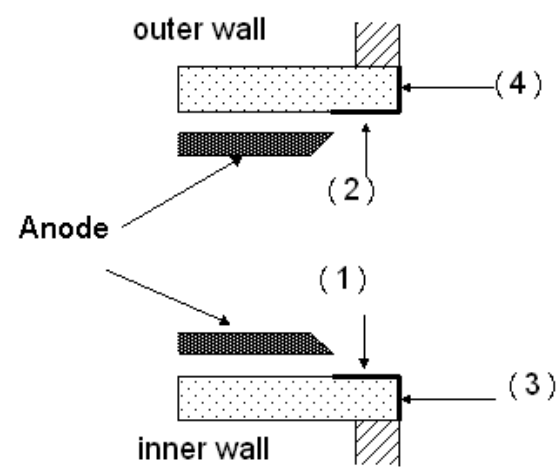

Figure 5 Schematic of thruster acceleration channel. Erosion is from surfaces labeled 1-4.

From the point of view of sensitivity of related experiments and required measurement times it is useful to consider the sensor sensitivity. The standard deviation of the ring-down time with empty cavity (laser detuned from resonance) is less than $1 \%$ which, for mirror reflectivity of $\mathrm{R} \sim 99.99 \%$, corresponds to an absorbance detection limit of $\sim 1 \mathrm{ppm}\left(10^{-6}\right)$ for a single ring-down acquisition. The measurement acquisition rate is approximately $200 \mathrm{~s}^{-1}$ yielding an absorption sensitivity of $\sim 1 \times 10^{-9} \mathrm{~cm}^{-1} \mathrm{~Hz}^{-1 / 2}$ based on detection over the full cavity length. Equivalently, in 1-s one can detect an absorption of 
$\sim 7 \times 10^{-9} \mathrm{~cm}^{-1}$ present over a $10 \mathrm{~cm}$ path length. The aforementioned values were measured without thruster operation. Operation of the thruster increased the noise level by a factor of $\sim 3$ owing to several factors including effects of vibration, heat-flux, and degradation of cavity mirror's reflectivity.

\section{Summary}

The CRDS sensor presented here contributes to filling the current gap in the ability to rapidly measure Hall thruster erosion for lifetime and contamination studies. The CRDS sensor allows high-sensitivity, quantitative, in situ measurements of sputtered particle number density. The versatility and fast time response of the sensor readily allows one to study effects of varying thruster operating conditions. In comparison to OES approaches, the use of CRDS, an absorption based technique, is attractive since ground states comprising a large fraction of the overall sputtered particle population can be directly measured. The demonstrated cw-CRDS sensor used a diode laser at $403 \mathrm{~nm}$ to probe manganese atoms sputtered from the stainless steel acceleration channel of a $1 \mathrm{~kW}$ class anode layer-type Hall thruster. The optical cavity was fiber coupled in and out of the vacuum chamber and measures were taken to combat mirror contamination and thermal effects that can degrade CRDS sensitivity. The flexibility of such an approach makes it amenable to implementation in different vacuum chamber facilities.

We have made a study on the dependence of erosion rate on discharge voltage and found a relatively linear dependence. A similar trend was observed in accompanying mass loss tests. For quantitative comparison of the CRDS and mass-loss we have employed a finite-element model based on the sputtering conditions and geometry. Ultimately this kind of model should be replaced by more sophisticated approaches, but for the purpose of demonstrating the technique, the agreement between the directly measured mass erosion rate and that determined from the model (using the CRDS), and between the measured and modeled CRDS lineshape, is taken as validation of the sensor. In order to more fully estimate the erosion rate, one should measure along several optical axes displaced perpendicularly relative to the thruster axis in order to estimate the radial density distribution of sputtered particles using Abel inversion approaches. Relating the CRDS number density to flux or erosion rate also requires knowledge of the particle velocity which may be determined more precisely from CRDS absorption lineshapes or complementary LIF measurements. On the other hand, even in the absence of more detailed measurements and modeling, the high time response and in situ nature of the CRDS can be of great utility in understanding erosion rate trends, i.e. monitoring how the erosion signal varies with changes in thruster operating conditions. In either case, the cw-CRDS sensor provides a new and powerful tool for sputter erosion and lifetime studies. Indeed, ongoing work involves application of cw-CRDS for study of boron nitride insulator channels [27]. 


\section{Acknowledgements}

The authors thank Prof. Alec Gallimore for collaboration in this enterprise and thank the Japan Society for the Promotion of Science, Japan for their financial support through a Grant-in-Aid for Scientific Research (S), No. 16106012,

\section{Reference}

[1] Kaufman, H. R., “Technology of Closed-Drift Thrusters,” AIAA Journal, 0001-1452, Vol. 23, No. 1, 1985, pp. 78-87. doi: $10.2514 / 3.8874$

[2] Kim, V., "Main physical features and processes determining the performance of stationary plasma thrusters," Journal of Propulsion and Power, Vol. 14, No. 5, 1998, pp. 736-743.

doi: $10.2514 / 2.5335$

[3] Choueiri, E. Y., "Fundamental difference between the two Hall Thruster Variants," Physics of Plasmas, Vol. 8, No. 11, 2001, pp. 5025-5033.

[4] Garner, C. E., Polk, J. E., Goodfellow, K. D., and Brophy, J. R., "Performance Evaluation and Life Testing of the SPT-100,” Proceedings of the 23rd International Electric Propulsion Conference, IEPC paper 93-091, Sept. 1993,

[5] Garner, C. E., Brophy, J. R., Polk, J. E., and Pless, L. C. “A 5730-Hr Cyclic Endurance Test of the SPT-100,” AIAA paper, 95-2667, July 1995.

[6] Jacobson, D. and Manzella, D., “High Voltage TAL Erosion Characterization,” AIAA paper 2002-4257, July, 2002.

[7] Yamamoto, N., Yokota, S., Matsui, M., Komurasaki and K., Arakawa, Y., "Measurement of Erosion Rate by Absorption Spectroscopy in a Hall Thruster,” Review of Scientific Instruments, Vol. 76, No. 8, 083111, 2005

doi:10.1063/1.2001630

[8] Cheng, Y. S. and Martinez-Sanchez, M., "Hybrid Particle-in-Cell Erosion Modeling of Two Hall Thrusters," Journal of Propulsion and Power, Vol. 24, No. 5, 2008, pp. 987-998.

doi: $10.2514 / 1.36179$

[9] Eagle, W. E,. , Boyd,I. D., Trepp, S. G. and Sedwick, R. J., “The Erosion Prediction Impact on Current Hall Thruster Model Development,” AIAA paper -2008-5087, July, 2008.

[10] Sommier, E., Allis, M. K., Gascon, N., and Cappelli, M. A., "Wall Erosion in 2D Hall Thruster Simulations," AIAA Paper 2006-4656, July 2006.

[11] Yalin, A. P., Surla, V., Farnell, C., Butweiller, M., and Williams, J. D., "Sputtering studies of multi-component materials 
by weight loss and cavity ring-down spectroscopy,” AIAA paper 2006-4338, July, 2006.

[12] Tsuge, H. and Esho, S., “Angular distribution of sputtered atoms from polycrystalline metal targets,” Journal of Applied Physics, Vol. 52, Issue 7, 1981, pp. 4391-4395.

doi: $10.1063 / 1.329365$

[13] Yalin, A. P., Williams, J. D., Surla, V. and Zoerb, K. A., "Differential Sputter Yield Profiles of Molybdenum due to Bombardment by Low Energy Xenon Ions at Normal and Oblique Incidence,” Journal of Physics D: Applied Physics,Vol. 40, 2007, pp. 3194-3202.

doi: 10.1088/0022-3727/40/10/025

[14] Mannami, M., Kimura, K., and Kyoshima, A., “Angular distribution measurements of sputtered Au atoms with quartz oscillator microbalances,” Nuclear Instruments and Methods, Vol. 185, No. 1-3, 1981, pp.533-537 doi: 10.1016/0029-553X(81)91252-0

[15] Kundu, S., Ghose, D., Basu, D., and Karmohapatro, S. B., “The angular distribution of sputtered silver atoms,” Nuclear Instruments and Methods in Physics Research Section B, Vol. 12, No. 3, 1985, pp.352-357. doi: 10.1016/0168-583X(85)90033-3

[16] Wucher, A. and Reuter, W., “Angular distribution of sputtered particles from metals and alloys,” Journal of Vacuum Science and Technology A,Vol. 6, No. 4, July, 1998, pp.2316-2318.

[17] Mantenieks, M., Foster, J., Ray P., Shutthanandan, S., and Thevuthasan, T., “Low energy xenon ion sputtering yield measurements," Proceedings of the 27th International Electric Propulsion Conference, IEPC paper 01-309, Electric Rocket Propulsion Society, Oct. 2001.

[18] Andersen, N., B. Andresen, and Veje, E., “Atomic excitations in sputtering processes,” Radiation Effects and Defects in Solids, Vol. 60, No. 1/4, 1982, pp.119-127.

[19] Doerner, R.P., Whyte, D.G., and Goebel, D.M., "Sputtering yield measurements during low energy xenon plasma bombardment,” Journal of Applied Physics, Vol. 93, No. 9, 2003, pp.5816-5823.

doi: $10.1063 / 1.1566474$

[20] Pellin, M.J., Wright, R.B., and Gruen, D.M., “Laser fluorescence spectroscopy of sputtered zirconium atoms,” Journal of Chemical Physics, Vol. 74, No. 11, 1981, pp.6448-6457

doi: $10.1063 / 1.440983$

[21] Nicolussi, G., Husinsky, W., Gruber, D. and Betz, G.,"Formation of metastable excited Ti and Ni atoms during sputtering,” Physical Review B, Vol. 51, No. 14, 1995, pp.8779-8788. 
doi: 10.1103/PhysRevB.51.8779

[22] Goehlich, A., "Investigation of time-of-flight and energy distributions of atoms and molecules sputtered from oxygen-covered metal surfaces by laser techniques,” Applied Physics A, Vol. 72, 2001, pp.523-529.

[23] Surla,V. Wilbur, P.J. Williams, J.D. Johnson,M. Yalin, A.P., “Sputter Erosion Measurements of Titanium and Molybdenum by Cavity Ring-Down Spectroscopy,” Review of Scientific Instruments, Vol. 75, No. 9, 2004, pp. 3025-3030. doi: $10.1063 / 1.1786354$

[24] Yalin, A.P., Surla, V., Butweiller, M., Williams, J.D. “Detection of Sputtered Metals using Cavity Ring-Down Spectroscopy,” Applied Optics, Vol. 44, No. 30, 2005, pp. 6496-6505.

doi:10.1364/AO.44.006496

[25] Yalin, A.P., Surla, V. "Determination of Number Density and Velocity of Sputtered Particles by Cavity Ring-Down Spectroscopy,” Proceedings of the 29th International Electric Propulsion Conference, IEPC paper 2005-300, Oct. 2005.

[26] Yalin, A.P., Surla, V. “Velocity Measurements by Cavity Ring-Down Spectroscopy,” Optics Letters, Vol.30, 2005, pp. 3219-3221.

doi:10.1364/OL.30.003219

[27] Yalin, A. P., Tao, L., Sullenberger, R., Oya M., Yamamoto, N., Gallimore, A., and Smith, T. ” High-Sensitivity Boron Nitride Sputter Erosion Measurements by Continuous-Wave Cavity Ring-Down Spectroscopy,” Proceedings of the 44th AIAA/ASME/SAE/ASEE Joint Propulsion Conference and Exhibit, AIAA paper 2008-5091, July, 2008.

[28] Surla, V. and Yalin, A. P., "Differential sputter yield measurements using cavity ring-down spectroscopy,” Applied Optics, Vol. 46, Issue 19, 2007, pp. 3987-3994.

doi:10.1364/AO.46.003987

[29] Bugrova, A.I., Bishaev, A.M., Desyatskov, A.V., Kozintseva, M.V. and Prioul, M., "Spectral Inverstigation of SPT MAG Insulator Erosion,” Proceedings of the 29th International Electric Propulsion Conference, IEPC paper 2005-167, Oct. 2005

[30] Dyshlyuk, E.N. and O.A. Gorshkov, “Spectroscopic Investigation of a Hall Thruster Ceramic Acceleration Channel Erosion Rate,” Proceeding of the 42nd AIAA Joint Propulsion Conference and Exhibit, AIAA 2006-4660, July, 2006

[31] Karabadzhak, G.F., Semenkin, A.V., Solodukhin, A.E. and O.S.Tverdokhlebov, "Evaluation of Impurity Composition and Content in the TAL at Various Operating Regimes," Proceedings of the 29th International Electric Propulsion Conference, IEPC paper 2005-147, Electric Rocket Propulsion Society, Oct., 2005.

[32] Pagnon, D., Lasgorceix, P., and Touzeau, M., "Control of the ceramic erosion by optical emission spectroscopy: results 
of PPS1350-G measurements,” Proceedings of the 4th International Spacecraft Propulsion Conference (ESA SP-555), June, 2004.

[33] Tverdokhleboc, O. S. and Karabadzhak, “TAL relative erosion rate real-time measurements through analysis of its emission spectra,” Proceedings of the 28th International Electric Propulsion Conference, IEPC paper 023-0336, March, 2003.

[34] Leray, P., Bonnet, J., Pigache, D., Minea, T., Bretagne, J. and Touzeau, M., “Spatially resolved emission spectroscopy along a SPT channel interpretation of data by a collisional-rediative model,” Proceedings of the 25th International Electric Propulsion Conference, IEPC paper 97-054, Aug. 1997.

[35] Domonkos, M. T., and Stevens, R. E., “Assessment of spectroscopic real-time ion thruster grid erosion-rate measurement" Proceedings of the 36th AIAA/ASME/SAE/ASEE Joint Propulsion Conference and Exhibit, AIAA paper 2000-3815, July, 2000

[36] McWhirter, R.W.P., "Spectral Intensities," Plasma Diagnostic Techniques, edited by Huddlestone, R.H., and Leonard, S.L., 1st ed., Academic Press Inc., New York, 1965, pp. 202-214

[37] Semenkin, A., "Investigation of Erosion in Anode Layer Thrusters and Elaboration High Life Design Scheme," Proceedings of the 23rd International Electric Propulsion Conference, IEPC Paper 93-231, Sept. 1993.

[38] Popov, Iu. S., and Zolotaikin, Iu. M., “Effect of Anomalous Conductivity on the Structure of the Anode Sheath in a Hall Current Ion Source,” Soviet Journal of Plasma Physics, Vol. 3, No.2, Mar.-Apr., 1977 pp. 210-213.

[39] Yamamoto, N., Komurasaki, K., and Arakawa, Y., “Discharge Current Oscillation in Hall Thrusters,” Journal of Propulsion and Power, Vol. 21, No. 5, 2005, pp. 870-876.

doi: $10.2514 / 1.12759$

[40] Busch K. W. and Busch M.A., “Introduction to Cavity-Ringdown Spectroscopy,” Cavity-Ringdown Spectroscopy, American Chemical Society, Washington DC, 1999, pp.7-19.

[41] Berden, G., Peeters, R. and Meijer, G., "Cavity Ring-Down Spectroscopy: Experimental Schemes and Applications.” International Reviews in Physical Chemistry, Vol. 19, No. 4, Oct., 2000 , pp. 565 - 607 doi: 10.1080/014423500750040627

[42] Paldus, B. A. and Kachanov, A. A., “An historical overview of cavity enhanced methods ” Canadian Journal Physics, Vol. 83, Oct. 2005, pp. 975-999.

[43] Zalicki, P. and Zare, R.N., “Cavity ring-down spectroscopy for quantitative absorption measurements,” Journal of Chemical Physics, Vol. 102, No. 7, 1995, pp. 2708-2717. 
[44] Yalin, A.P., and Zare, R.N.” Effect of Laser Lineshape on the Quantitative Analysis of Cavity Ring-Down Signals,” Laser Physics,” Vol. 12, No. 8, 2002, pp. 1065-1072.

doi: $10.1063 / 1.468647$

[45] Holloway, J. P., Shannon, S., Sepke, S. M., and Brake, M. L., “A reconstruction algorithm for a spatially resolved plasma optical emission spectroscopy sensor,” Journal of Quantitative Spectroscopy and Radiative Transfer, Vol. 68, No. 1, 2001, pp.101-115

doi:10.1016/S0022-4073(00)00017-0

[46] Takahashi, N., Yamamoto, N., Nakashima, H., Yokota, S., Matsui, M., Komurasaki, K. and Arakawa, Y, “Investigation of Internal Plasma Structure in an Anode-layer Hall Thruster,” Proceedings of the 30th International Electric Propulsion Conference, IEPC Paper 2007-092, Sept. 2007.

[47] Tao, L., Yamamoto, N. and Yalin, A. P., "Cavity Ring-Down Spectroscopy Sensor for Ion Beam Etch Monitoring and End-Point Detection of Multilayer Structures," Review of Scientific Instruments, Vol. 79, 2008, 115107.

doi: 10.1063/1.2995765

[48] Booth, A. J., Shallis, M. J. and Wells, M., "Hyperfine structure measurements for lines of astrophysical interest in MN I,” Royal Astronomical Society, Monthly Notices, Vol. 205, Oct. 1983, pp. 191-205.

[49] Yokota, S., Matsui, M., Sako, D., Yamamoto, N., Koizumi, H., Komurasaki, K., Nakashima, H. and Arakawa, Y., “Diagnostics of Hall Thruster Plume by Laser Absorption Spectroscopy” Proceedings of the 42th AIAA/ASME/SAE/ASEE Joint Propulsion Conference and Exhibit, AIAA paper 2006-5028, July, 2006

[50] Gryzinski, M., “Classical theory of atomic collisions. I. Theory of inelastic collisions,” Physical Review, Vol. 138, No. 2A, April, 1965, pp. 336-358

doi: 10.1103/PhysRev.138.A336 